\title{
Thinning Grayscale Well-Composed Images: A New Approach for Topological Coherent Image Segmentation
}

\author{
Jocelyn Marchadier, Didier Arquès, and Sylvain Michelin \\ Université de Marne-la-Vallée, Equipe Image, Institut Gaspard Monge, \\ 5, Boulevard Descartes, 77454 Champs sur Marne Cedex \\ Jocelyn.Marchadier@univ-mlv.fr
}

\begin{abstract}
Usual approaches for constructing topological maps on discrete structures are based on cellular complexes topology. This paper aims to construct a coherent topological map defined on a square grid from a watershed transformation. We propose a definition of well-composed grayscale images based on the well-composed set theory and the cross-section topology. Properties of two different thinning algorithms are studied within this scope, and we show how to obtain a thin crest network. We derive an efficient algorithm that permits the construction of a meaningful topological map. Finally, we demonstrate the usefulness of this algorithm for multilevel image segmentation.
\end{abstract}

Keywords. Topological map, thinning, well-composed images.

\section{Introduction}

A digital image may be seen as the digitalization of a piecewise continuous function. The discontinuities of the underlying continuous function are of primary importance in many shape recognition processes, as they usually describe the shapes of objects appearing on an image. A piecewise continuous function may be represented by a topological map, which can be viewed as a partition of the plane into three sets of points: a finite set $S$ of points, a finite set $A$ of disconnected Jordan arcs having elements of $S$ as extremities, and a set of connected domains, the faces, which boundaries are unions of elements of $S$ and $A$. The definition and the extraction of a topological map from a digital grayscale image coherent with the one describing the underlying continuous function would be a major achievment. Although such a structure can been defined straightforwardly on an hexagonal grid, the square grid poses consistency problems.

The notion of discontinuity is lost once a function is digitalized. Segmentation may be viewed as a process that tries to catch the discontinuities of an underlying continuous function. Two main approaches to segmentation may be distinghished. The first approach consists of approximating the discrete function by a piecewise continuous function, and is usually referred to as a region oriented segmentation. The second approach tries to directly catch the discontinuities of an underlying continuous function using an heuristic provided by a

A. Braquelaire, J.-O. Lachaud, and A. Vialard (Eds.): DGCI 2002, LNCS 2301, pp. 360-371 2002. (C) Springer-Verlag Berlin Heidelberg 2002 
gradient operator defined on discrete functions, and is referred as contour oriented segmentation.

Recent works [47] aim to develop a coherent topological structure describing a digital image from the information provided by regions. The different structures described use a discrete topology based on the decomposition of the support domain of an image into three kind of elements of different dimensions, i.e. surface elements, associated with the discrete points of the support, edge elements, which are the edges seperating two surface elements, and vertices of the so defined grid 6,10]. On one hand, such a partition has nice topological properties, but on the other hand, it suffers from many practical drawbacks, such as the amount of memory needed to store the entire partition, and difficulties faced when trying to construct the partition from the contour information.

Alternatively, a topological partition of an image may be directly defined by a digital topology only involving points on the square grid. In order to face the connectivity paradox, several neighborhood systems are usually used together. This is done either by considering different adjacent relations for points belonging to a set and its complement [1], or by assigning different neighborhoods to each point of $Z^{2}$ in a data independent manner, which can be formally stated using the framework proposed in [9] (this framework can also be used in the cellular complex approach [10]).

Watersheds or more generally graytone skeletons can be used in this context to retrieve a vertex/arc network (crests lines) and faces (catchment basins) from a discrete topographic surface such that the modulus of the gradient viewed as a relief, which is exactly the seeked partition of the image. However, many consistency problems are encountered on a square grid. Approaches that work by suppressing points from a potential crests network (grayscale thinning) or by adding points to connected sets of points [1.3 do not usually guarantee that the extracted crests network is thin (figure 1). Thick configurations of crests pose

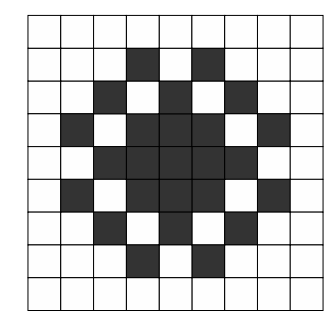

Fig. 1. Thick irreductible configuration of points [1]

obvious problems when one is trying to link points from the resulting crests network in order to obtain digital curves and vertices of the topological partition. Approaches that work by linking potential crest points [16[17], constructing a raster graph, do not usually guarantee that the faces defined by the cycles of the graph are composed of a unique connected component (figure 2). 


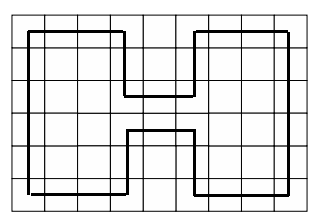

Fig. 2. Connected components assiciated with a cycle of a raster graph

Latecki and al. proposed to face the problem of thickness of skeletons on digital binary images by forbidding some configurations of points [15]. They propose a thinning operator that preserves the properties of the so called wellcomposed binary images, resulting in a thin skeleton. He also demonstrated a Jordan theorem that is verified on well-composed sets of points. He extended the property of well-composedness to multicolor images.

In this contribution, we first recall some classical notions of digital topology (section 2) and some properties of well-composed sets (section 3). We redefine grayscale well-composed images using the cross section topology formalism [2 3 , 16], adapt a grayscale thinning to well-composed graylevel images, and proove some of its properties (section 4). We derive an algorithm which construct a topological partition from an ultimate thinning of a well-composed image, and finally present an application (section 5 ).

\section{Digital Topology: Basic Notions}

A discrete image $I$ is a function from $Z^{2}$ to a set $E$. When $E=\{0,1\}, I$ is said to be a binary digital image. When $E=\{0, \ldots, k\}, I$ is said to be a grayscale image.

Two point $p_{1}=\left(x_{1}, y_{1}\right) \in \mathbb{Z}^{2}$ and $p_{2}=\left(x_{2}, y_{2}\right) \in \mathbb{Z}^{2}$ are

-4 -adjacent if and only if $d_{4}\left(p_{1}, p_{2}\right)=\left|x_{1}-x_{2}\right|+\left|y_{1}-y_{2}\right|=1$.

-8 -adjacent if and only if $d_{8}\left(p_{1}, p_{2}\right)=\max \left(\left|x_{1}-x_{2}\right|,\left|y_{1}-y_{2}\right|\right)=1$.

The n-neighborhood $\Gamma_{n}(p)$ of a point $p$ is the set of all the points n-adjacent to $p$, with $n=4$ or $n=8$. A n-connected path is an ordered set $C=\left\{p_{1}, \ldots, p_{m}\right\}$ such that for all the points $p_{i, 1<i \leq n}, p_{i}$ is n-adjacent to $p_{i-1}$. A n-connected simple curve $C$ is a n-connected path such that all the points of $C$ have at most two n-connected neighbors in $C$. A n-connected simple closed curve $C=$ $\left\{p_{1}, \ldots, p_{m}\right\}$ is a n-connected simple curve such that $p_{1} \in \Gamma_{n}\left(p_{m}\right)$. A set $S \subset$ $\mathbb{Z}^{2}$ is n-connected iff $\forall\left(p_{1}, p_{2}\right) \in S^{2}, \exists C \subset S^{2}, C$ is a n-connected path and $\left(p_{1}, p_{2}\right) \in C$. A n-connected component of a set $S \subset Z^{2}$ is a subset $C$ of $S$ such that $\forall p_{1} \in C, \forall p_{2} \in S, p_{2} \in \Gamma_{n}\left(p_{1}\right) \Rightarrow p_{2} \in C$.

\section{Well-Composed Sets of Points}

A coherent topological structure of a digital image should respect an equivalent of the Jordan curve theorem by which the complement of a simple closed curve 
is a set composed of two connected components. One of the major drawbacks of digital topology is known as the connectivity paradox. If the same neighborhood system is used for studying the connectivity of a set and its complement, the Jordan curve theorem does not have its digital counterpart on a squared grid. In order to solve this problem, two neighborhood systems ( 8 and 4-neighborhood) are used together, defining the connectivity of respectively a set and its complement 11. Latecki and al. 15 proposed a different approach. By forbidenning some local configurations of the studied sets of points, one can use a coherent 4-neighborhood system. In this section, we present the results that they obtained which are usefull for the rest of this article. Readers interested in the demonstrations of those results may refer to 1315 .

A subset $S$ of $Z^{2}$ is weakly well-composed iff each 8-connected component of $S$ is a 4 -connected component of $S$. A subset $S$ of $Z^{2}$ is well-composed iff $S$ and $\bar{S}$ are weakly well-composed. The figure 3 illustrates the concept of wellcomposedness. On the left hand side of the drawing, the set of gray points form a weakly well-composed set of points, while the right hand side drawing shows a set of points which is well-composed. In order for a set of points to be wellcomposed, configurations like the one surrounded by a bold square are forbidden. Thus, well-composed sets can be characterized by local configurations.

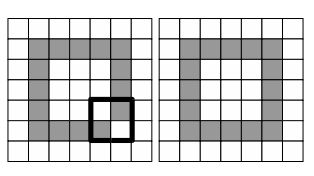

Fig. 3.

This leads to the statement of a new Jordan theorem on well composed set:

Theorem 1. The complement of a well-composed simple closed curve is formed by two well-composed connected components.

Classical thinning algorithms do not usually conserve the property of wellcomposedness. However, by slightly modifying the definition of simple points, we obtain a thinning that conserves well-composedness.

A point $p$ is simple for a well-composed set $S \subset Z^{2}$ iff there exists a bijection between 4-connected components of $S \cup\{p\}$ and 4-connected components of $S \backslash\{p\}$, and between 4-connected components of $\bar{S} \cup\{p\}$ and 4-connected components of $\bar{S} \backslash\{p\}$. Simple points for well-composed sets can be characterized by a few local configurations. One can show that a point $p$ is simple for a well-composed set $S$ iff there exists exactly one 4-connected component of $S$ 4 -adjacent to $p$ and one 4-connected component of $\bar{S} 4$-adjacent to $p$.

A thinning of a well-composed set $S$ is a set $S^{\prime}$ obtained from $S$ by iteratively deleting simple points of $S$. By construction, a thinning of a well-composed set is a well-composed set. 
A set $S$ is irreductible iff no point of $S$ is simple. Let the n-interior of a set $S$ of points be the set $n-\operatorname{int}(S)=\left\{p \in S, \Gamma_{n}(p) \subset S\right\}$.

An irreductible thinning of a well-composed set is thin, which can be formally stated as:

Theorem 2. The 8-connected components of the 4-interior of an irreductible well-composed set are reduced to one point.

The proof of this theorem found in [13] holds for the earlier definition of simple points. This can be used to enumerate all the irreductible local configurations (figure 44). On this figure, white lines show how to link points from an irreductible well-composed set of points in order to obtain a vertex/arc network defining a coherent topological partition.

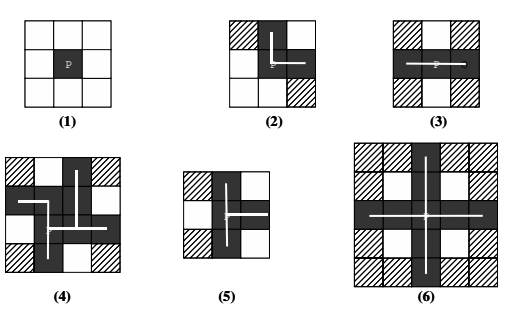

Fig. 4. Local irreductible configurations for well-composed sets

\section{Well-Composed Grayscale Images}

Meyer [16] proposed to characterize crest points on a grayscale image by studying a familly of binary images, which are obtained by thresholding the original image by all possible threshold values. Crest points are the points that change the homotopy of one of the binary images. More recently, cross-section topology has been introduced by Bertrand and al. [2,3]. It has led to the development of an efficient watershed algorithm [5]. The definition of well-composed multicolor images has also been proposed 12 .

In this section, we use the definition of well-composed grayscale images [14], which is compatible with the cross section topology. We then study the properties of thinnings of well-composed grayscale images.

Let $I$ be a grayscale digital image, defined as a function from $Z^{2}$ to $E=$ $\{1, \ldots, m\}$.

A c-section of $I$ is the set of points $F_{c}=\left\{(x, y) \in \mathbb{Z}^{2}, I(x, y) \geq c\right\}$.

A c-cut of $I$ is the set of points $E_{c}=\left\{(x, y) \in \mathbb{Z}^{2}, I(x, y)=c\right\}$

A grayscale digital image $I$ is said to be well-composed iff all its c-sections are well-composed sets. The critical configurations depicted on figure 5 are forbidden for well-composed grayscale images. 


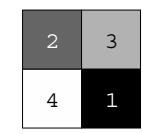

Fig. 5. Forbiden configurations for well-composed grayscale images. Dark points are points having highest values of $I$.

A point $p \in Z^{2}$ is said to be destructible iff it is simple for the section $F_{c}$, with $c=I(p)$.

We define the two sets $\Gamma^{+}(p)=\left\{p^{\prime} \in \Gamma_{8}(p), I\left(p^{\prime}\right) \geq I(p)\right\}$ and $\Gamma^{-}(p)=$ $\left\{p^{\prime} \in \Gamma_{8}(p), I\left(p^{\prime}\right)<I(p)\right\}$.

A destructible point $p$ verifies that both $\Gamma^{+}(p)$ and $\Gamma^{-}(p)$ are composed of a unique 4-connected component, with a point 4-adjacent to $p$.

The destruction of a point $p$ is the operation consisting of substracting 1 from $I(p)$.

An image $I^{\prime}$ is a thinning of a grayscale well-composed image $I$ iff it can be obtained from $I$ by iteratively destructing destructible points from $I$.

Theorem 3. Let I be a well-composed grayscale image, and $I^{\prime}$ be a thinning of I. Then $I^{\prime}$ is a well-composed grayscale image.

This can be demonstrated by observing that if $I^{\prime}$ is a thinning of $I$, then a c-section of $I^{\prime}$ is, consequently to the definition of a destructible point, a thinning of the c-section of $I$, independently of the value of $c$. All the c-sections of $I$ are well-composed. As a thinning of a well-composed set is well-composed, all the c-sections of $I^{\prime}$ are well-composed, thus $I^{\prime}$ is well-composed.

An graylevel well-composed image $I$ is irreductible iff no point of $I$ is destructible.

We define a plateau of a well-composed image $I$ as a 4-connected set $S$ such that $\forall\left(p, p^{\prime}\right) \in S^{2}, I(p)=I\left(p^{\prime}\right)$. A regional minimum $S$ is a plateau such that $\forall p \in S, \Gamma_{4}(p) \cap \bar{S} \neq \emptyset, \forall p^{\prime} \in \Gamma_{4}(p) \cap \bar{S}, I\left(p^{\prime}\right)>I(p)$.

Theorem 4. The set composed of all the points belonging to regional minima of a well-composed image I is well-composed.

Let $p$ and $p^{\prime}$ be two 8-adjacent but not 4-adjacent points in regional minima of $I$. Suppose that $\Gamma_{4}(p) \cap \Gamma_{4}\left(p^{\prime}\right)$ is not included in a regional minimum of $I$. Then $I$ is not well-composed, since this construction is forbidden for well-composed images. This leads to a contradiction.

Theorem 5. There exists a bijection between regional minima of a wellcomposed image I and regional minima of a thinning of $I$.

We consider a thinning $I^{\prime}$ of a well-composed image $I$. The points belonging to a regional minimum of a well-composed set $I$ are not destructible. If a point 
of a regional minimum of $I$ is destructible, then it is 4-adjacent to at least one point with a lower value of $I$, and thus, it is not a point of a regional minimum. The regional minima of $I$ are then subsets of regional minima of $I^{\prime}$ of $I$.

Let's suppose that a regional minimum $S^{\prime}$ of $I^{\prime}$ contains several regional minima of $I$. Let $c$ be the value of the highest regional minimum $S$ of $I$ such that $S \subset S^{\prime}$. The set $\overline{F_{c+1}} \cap S^{\prime}$ contains at least two 4-connected components bounded by points adjacent to $S$ (Jordan theorem). Thus, $\overline{F_{c+1}}$ contains more connected components than the set $\overline{F_{c+1}^{\prime}}$, where $F_{c+1}^{\prime}$ is the $(c+1)$-section of $I^{\prime}$. $I^{\prime}$ is not a thining of $I$, which contradicts the hypotheses.

The complementary sets of regional minima are arbitrarely thick. This is partly due to configurations like the one depicted on the figure 6 On this figure,

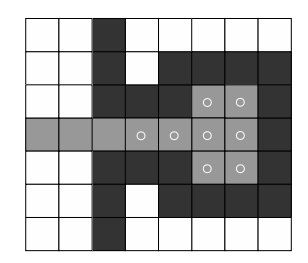

Fig. 6. Regional quasi-minimum

the set composed of the points marked by a circle can be used to define an interseting face of a topological partition.

We define a regional quasi-minimum as a 4-connected component of the set $Q=\left\{p \in \mathbb{Z}^{2}, \Gamma^{-}(p)=\emptyset\right\}$ defined on $I$. Note that a regional minimum is a regional quasi-minimum.

The set $Q$ defined on an irreductible well-composed image is not generally well-composed. For example, on the figure 17, $Q$ is composed of all the points marked by a circle, and $Q$ is not well-composed. This is due to the presence of peaks, i.e. points without upper neighbors. This leads to the following devlopments.

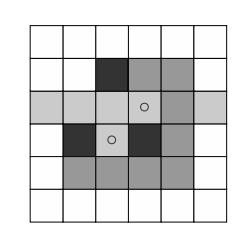

Fig. 7.

A leveling of an image $I$ [3] is a graylevel image obtained from $I$ by iteratively destructing destructible points and peaks from $I$. 
Theorem 6. Let I be a graylevel well-composed image, and let $I^{\prime}$ be a leveling of $I$. Then $I^{\prime}$ is well-composed.

The destruction of a destructible point from a graylevel well-composed image $I$ will result in a thinning of $I$ which is well-composed (c.f. theorem [3). Now consider a peak $p$ from a well-composed image $I$. Let $p^{\prime}$ one of its neighbors such that $\forall p^{\prime \prime} \in \Gamma_{8}(p) I\left(p^{\prime \prime}\right) \leq I\left(p^{\prime}\right)$. As $p$ is a peak, we have $I(p)>I\left(p^{\prime}\right)$. Let $I^{\prime}$ the image obtained by destructing $p$ from $I$. Then $I^{\prime}(p) \geq I^{\prime}\left(p^{\prime}\right)$, and the ordering of the other points from $\Gamma_{8}(p)$ is not changed. Thus, we do not create a forbiden configuration for well-composed graylevel images, and $I^{\prime}$ is well-composed.

As the two operations are conserving well-composedness, their iterative application on a well-composed image will yield in a well-composed image.

By applying the leveling transformation on a well-composed graylevel image until stability, we obtain a well-composed irreductible graylevel image such that none of its point is a peak. On such images, regional quasi-minima are wellcomposed.

Theorem 7. Let I be an irreductible well-composed graylevel image such that no point of $I$ is a peak. The set $Q=\left\{p \in \mathbb{Z}^{2}, \Gamma^{-}(p)=\emptyset\right\}$ defined on $I$ is well-composed.

Let $I$ be a well-composed grayscale image. Let $p$ and $p^{\prime}$ be two points 8-adjacent, but not 4-adjacent, belonging to the set $Q=\left\{p \in \mathbb{Z}^{2}, \Gamma^{-}(p)=\emptyset\right\}$, such that the two points $p_{1}$ and $p_{2} 4$-adjacent to $p$ and $p^{\prime}$ are not belonging to $Q$. Necessarly, $I(p)=I\left(p^{\prime}\right), I\left(p_{1}\right) \geq I(p)$ and $I\left(p_{2}\right) \geq I(p)$. Moreover, as $I$ is well-composed, $I\left(p_{1}\right)=I(p)$ or $I\left(p_{2}\right)=I(p)$. Let's suppose, without loss of generality, that $I\left(p_{1}\right)=I(p)$, which corresponds to the figure 8

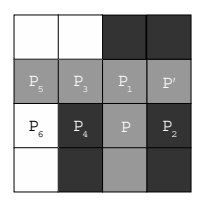

Fig. 8.

$I\left(p_{3}\right) \geq I(p)$ by hypothese. $I\left(p_{3}\right)=I(p)$ otherwise $p_{3}$ is either destructible or $I$ is not well-composed. $I\left(p_{5}\right) \geq I\left(p_{3}\right)$ and $I\left(p_{6}\right)<I\left(p_{3}\right)$ otherwise $p_{3}$ is destructible again. Then, $p_{4}$ is destructible, which leads to a contradiction.

We can now expose the following result, which states that each cut of an irreductible graylevel image without peak is thin.

Theorem 8. Let I be an irreductible well-composed graylevel image from $\mathbb{Z}^{2}$ to a set $E$, such that no point of $I$ is a peak, and the set $Q=\left\{p \in \mathbb{Z}^{2}, \Gamma^{-}(p)=\emptyset\right\}$ defined on $I$. For all $c \in E$, the 8-connected components of the 4-interior of the set $E_{c} \backslash Q$ are composed of at most one point, where $E_{c}$ is the c-cut of $I$. 
Let $c \in E$. Consider a point $p$ with $\Gamma^{-}(p) \neq \emptyset$ and $I(p)=c$. If $\left\{p^{\prime} \in\right.$ $\left.\Gamma_{8}(p), I\left(p^{\prime}\right)>I(p)\right\} \neq \emptyset$, then the c-cut of $I$ respect one of the local configurations of the irreductible well-composed sets depicted on figure 4 We now consider two points $p$ and $p^{\prime}$ belonging to the same c-cut, 8-adjacent to each other, such that $p$ is adjacent to at least one point of lower value and one point of upper value. Thus, one of the local configurations depicted on figure 9 holds.

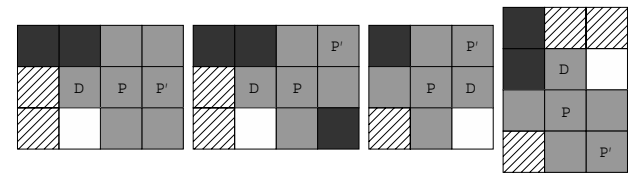

Fig. 9.

The point noted $D$ is destructible on these configurations and consequently $I$ is not irreductible.

\section{Constructing a Topological Partition and Application}

The topological map defined on the digital plane is composed of:

- a set $S$ of points which are vertices of the map,

- a set $A$ of arcs, which are 4-connected disconnected digital curves, of which the extremities are elements of $S$,

- a set $F$ of faces, which are 4-connected sets of points, of which the boundaries are elements of $S$ and $A$.

Such a structure can be built from an irreductible well-composed grayscale image without peak, obtained by a leveling transformation of a well-composed grayscale image. The faces of the topological partition are the regional quasi-minima. The vertex/arc network can be retrieved by linking points that have at least one lower neighbor in the following way:

- Points belonging to the same c-cut are linked together using one of the configurations of the figure 4 .

- Points belonging to different c-cuts are linked according to one of the configurations of the figure 10.

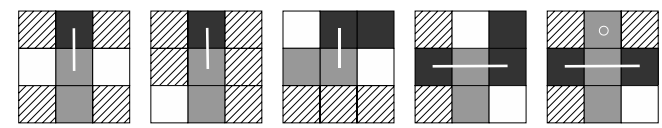

Fig. 10. 
We can prove, by reasoning on local configurations, that the cycles of the graph constructed as such correspond to regional quasi-minima. The figure 11 represents an irreductible well-composed image without peaks and its constructed vertex/arc network.

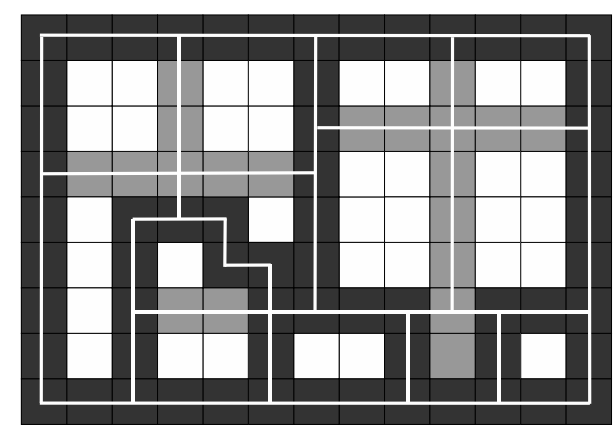

Fig. 11.

Different structures can be used to store this topological map, such as a combinatorial map [7] or a raster graph [17. Note that both structures can be obtained from an irreductible well-composed image without peak with a single pass algorithm. Moreover, the raster graph structure can be obtained with a parallel algorithm, as the linking process involves only local configurations.

The correspondance between the so defined map and the map that can be constructed from the linear discontinuities of the underlying piecewise continuous function is achieved when the topology of the interesting set of points is preserved under digitalization. Note also that the digitalization of a continuous curve is a digital well-composed curve [13. That motivates the use of the 4-neighborhood connectivity.

Figure 12 demonstrates the usefulness of this results for image segmentation. The first image of the figure is the original image. The second image is the irreductible well-composed image without peak that has been constructed from the modulus of a gradient of the original image. An algorithm, not detailed in this article, has been applied on the image of the modulus, turning it into a well-composed graylevel image. Thus, the second image is well-composed. The third image is the extracted crest network, where some arcs have been removed using a threshold strategy that was found to be stable and very fast on different kinds of images.

On all tested images, the crest network was found to be a thin set of points, although some thick configurations can be constructed. However, the linking is realized without ambiguity, and the topological map obtained in such a way have found to be usefull for shape recognition applications. 

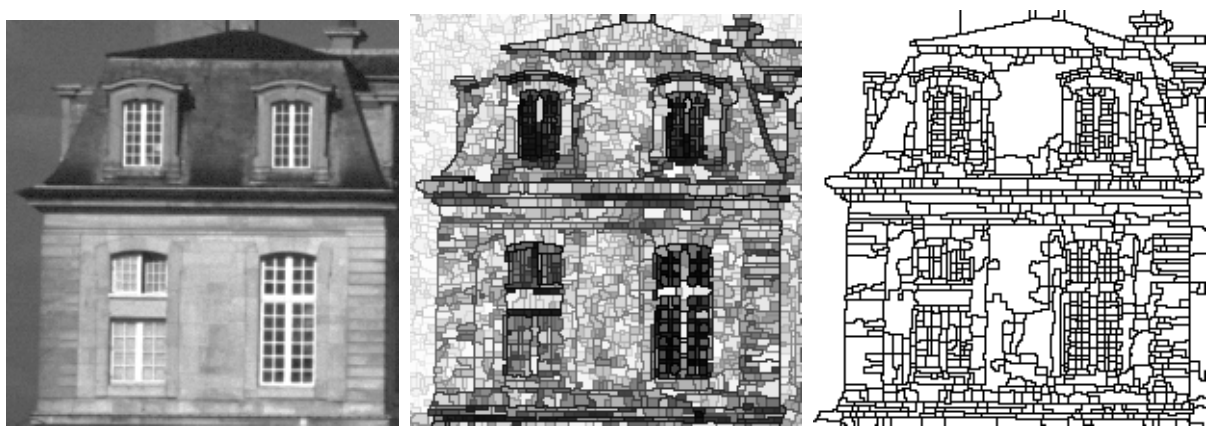

Fig. 12.

The definition of destructible points presented in this article is not well suited for non well-composed images. For such images, destructible points are defined with the classical 4/8-neighborhood system [3]. The thinning and leveling transformations defined with that system are resulting in images with drawbacks such that thick configurations of points (when the 8-neighborhood system is used to characterized crest points) or 8-connected isolated points.

\section{Conclusion}

In this contribution, we have used the cross section topology formalism in order to define a thinning operator that is internal to well-composed gray level images. Moreover, we have proposed a way to construct a topological map from the resulting thin image, and have shown that the obtained map is coherent in the sense that:

- a bijection exists between cycles of the map and 4-connected regions of the thin image,

- each Jordan arc of the map is a 4-connected digital curve.

The map can be constructed from a gray level image very efficiently, using previously published algorithms.

\section{References}

1. Arcelli C., Pattern Thinning by Contour Tracing, Computer Graphics and Image Processing, Vol. 17 (1981) 130-144

2. Bertrand B., Everat J.C., Couprie M., Topological approach to image segmentation, SPIE Vision Geometry V Proceedings, Vol. 2826 (1996)

3. Bertrand B., Everat J.C., Couprie M., Image segmentation through operators based upon topology, Journal of Electronic Imaging, Vol. 6(4) (1997) 395-405

4. Braquelaire J.-P., Brun L., Image Segmentation with Topological Maps and Interpixel Representation, Journal of Visual Communication and Image Representation, Vol. 9(1) (1998) 62-79 
5. Couprie, M., Bertrand G., Topological Grayscale Watershed Transformation, SPIE Vision Geometry V Proceedings, Vol. 3168 (1997) 136-146

6. Fiorio C., Approche interpixel en analyse d'images, une topologie et des algorithmes de segmentation, PhD Dissertation, Université de Montpellier, France, (1995) 198 pages.

7. Fiorio C., A topologically Consistent Representation for Image Analysis: the Frontiers Topological Graph, DGCI'96, Lectures Notes in Computer Sciences, no. 1176, (1996) 151-162

8. Gangnet M., Hervé J.-C., Pudet T., Van Tong J.-M., Incremental Computation of Planar Maps, SIGGRAPH Proc., Computer Graphics, Vol. 23(3) (1989) 345-354

9. Khalimsky E., Kopperman R., Meyer R., Computer Graphics and Connected Topologies on Finite Ordered Sets, Topology and its Applications, Vol. 36 1-17

10. Kovalevsky V. A., Finite Topology as Applied to Image Analysis, Computer Vision, Graphics and Image Processing, Vol. 46 141-161

11. Kong T. Y., Rosenfeld A., Digital Topology, Introduction and Survey, Computer Vision, Graphics, and Image Processing, Vol. 48 (1989) 357-393

12. Latecki L., Multicolor Well-Composed pictures, Pattern Recognition Letters, Vol. 16 (1995) 425-431

13. Latecki L., Discrete Representation of Spatial Objects in Computer Vision, Computational Imaging and Vision Vol. 11, Kluwer Academic Publishers (1999) 216 pages

14. Latecki L., Well-Composed Sets, Advances in Imaging and Electron Physics Vol. 112, Academic Press (2000) 95-163

15. Latecki L., Eckhardt U., Rosenfeld A., Well-Composed Sets, Computer Vision and Image Understanding, Vol. 61 (1995) 70-83

16. Meyer F., Skeletons and Perceptual Graphs, Signal Processing, Vol. 16 (1989) 335-363

17. Pierrot Deseilligny M., Stamon G., Suen C., Veinerization: A New Shape Description for Flexible Skeletonization, IEEE Trans. on PAMI, Vol. 20(5) (1998) 505521 